\title{
Contribuições do Seis Sigma: estudos de caso em multinacionais
}

\author{
Adriana Barbosa Santos ${ }^{\mathrm{a}, *}$, Manoel Fernando Martins ${ }^{\mathrm{b}}$ \\ a,*adriana@ibilce.unesp.br, UNESP, Brasil \\ ${ }^{\text {b} m a n o e l @ p o w e r . u f s c a r . b r, ~ U F S C a r, ~ B r a s i l ~}$
}

\begin{abstract}
Resumo
A literatura sugere que o Seis Sigma trouxe contribuições sob vários aspectos, tanto no âmbito da gestão da qualidade como também da gestão estratégica. No entanto, ainda há certa escassez de informações sobre esse assunto no cenário organizacional brasileiro. Este artigo visa reduzir essa carência da literatura, mostrando uma visão prática sobre as contribuições decorrentes dos investimentos no Seis Sigma para empresas do Brasil. A relevância de requisitos essenciais - como conceito de qualidade; combinação das abordagens estatística e estratégica; sistematização metodológica; formação de especialistas; e gestão de projetos - é discutida tanto em âmbito teóricoconceitual como prático. 0 método do estudo de caso foi aplicado para obter o embasamento empírico da pesquisa. Os investimentos no Seis Sigma feitos por duas subsidiárias brasileiras de empresas multinacionais norte-americanas (uma de manufatura e uma de serviços) são apresentados e reforçam a relevância dos requisitos supracitados.
\end{abstract}

Palavras-chave

Seis Sigma. Gestão da qualidade. Projetos. Autopeças. Serviços.

\section{Introdução}

Desde o seu surgimento, há quase duas décadas, o programa Seis Sigma vem ganhando a apreciação das organizações como uma abordagem para melhoria da qualidade com impacto positivo no desempenho do negócio. Essa consideração deu notoriedade não apenas na indústria de manufatura, mas também em operações de serviços, mostrando que o Seis Sigma está inserido no âmbito das discussões estratégicas das organizações.

Segundo Mitchell (1992), quando foi difundido pela Motorola no final da década de 80 , o Seis Sigma tinha como propósito ser uma iniciativa voltada para o controle da qualidade total, com ênfase na satisfação dos clientes e na eliminação de erros e falhas nos processos produtivos. Desde então, o Seis Sigma evoluiu conceitualmente e garantiu mais abrangência, principalmente, no que tange às ações gerenciais e estratégicas necessárias para sua implementação. 0 enfoque em dados e fatos e os benefícios reportados sobre aumento da eficiência dos processos de produção; redução dos custos associados com inspeção, retrabalho, insatisfação dos clientes e desperdício; foco em processos; melhoria contínua; foco no cliente; mudança de cultura; redução drástica da variação; e comprometimento tornaram o tema atraente e suscitaram a curiosidade e questionamentos sobre sua contribuição efetiva (SULLIVAN, 1984; KLEFSJÖ et al., 2001; GOH; XIE, 2004; CORONAD0; ANTONY, 2002; SCHROEDER et al., 2002).

A literatura permite inferir que o Seis Sigma é um programa que trouxe contribuições em vários aspectos para incrementar as discussões sobre gestão da qualidade e gestão estratégica, a partir da percepção de que sua estrutura incluía requisitos essenciais como: conceito de qualidade; combinação das abordagens estatística e estratégica; sistematização metodológica para implementar projetos; formação de especialistas; e gestão de projetos. No entanto, ainda há certa escassez na literatura de trabalhos que tragam uma visão prática sobre a relevância destes requisitos para empresas brasileiras, principalmente, no contexto de processos de serviços. Neste artigo, objetivou-se melhorar essa visão por meio de evidências empíricas obtidas pela realização de dois estudos de caso com subsidiárias brasileiras de empresas multinacionais norte-americanas, sendo uma de manufatura e outra de serviços. 


\section{Seis Sigma: definição, benefícios e requisitos essenciais}

Seis Sigma vem se destacando tanto na literatura como nos ambientes de manufatura e serviços como sendo mais do que um programa de qualidade. Devido a sua abrangência, atualmente coexistem na literatura várias definições que assinalam tanto o direcionamento metodológico (focado em qualidade) como também o estratégico (focado no desempenho do negócio). Fundamentando-se numa análise das definições de Seis Sigma, Santos (2006) propôs uma definição que buscou congregar elementos-chave para uma visão mais completa de Seis Sigma:

- Seis Sigma é uma abordagem que impulsiona a melhoria do desempenho do negócio e a valorização da satisfação dos clientes, por meio do enfoque estratégico de gerenciamento; da aplicação do pensamento estatístico em todos os níveis de atividades; do uso de indicadores de desempenho; da utilização de uma metodologia sistematizada que integre técnicas variadas para se avaliar e otimizar processos; e da aprendizagem decorrente da capacitação e comprometimento das pessoas.

Após surgir no cenário da gestão da qualidade, destacando-se principalmente como uma iniciativa de melhoria que enfocava a medição, uso de métodos estatísticos, o trabalho desenvolvido por equipe especializada e a definição clara de uma meta de desempenho operacional, quantificada por meio de um padrão sigma para avaliar, principalmente, processos de manufatura (MITCHELL, 1992; McAFADDEN, 1993; TADIKAMALLA, 1994; BEHARA et al., 1995), o Seis Sigma passou a estar inserido num contexto mais amplo, sendo reconhecido como uma estratégia efetiva de se aumentar o desempenho do negócio (BLAKESLEE, 1999; HARRY; SCHROEDER, 2000; SANDERS; HILD, 2000; PANDE et al., 2001; McADAM; BAILIE, 2002; MOTWANI et al., 2004).

$\mathrm{Na}$ última década, os reflexos positivos da implementação do Seis Sigma também em operações de serviços têm sido notórios na literatura, os quais incluem aplicações nos segmentos de serviços hospitalares, instituições financeiras, serviços de transporte de cargas e passageiros, entre outros (DOES et al., 2002; BRETT; QUENN, 2005; ANTONY, 2006; CHAKRABARTY; TAN, 2007; NONTHALEERAK; HENDRY, 2008).

Em termos práticos, estudos com empresas brasileiras asseguram que o Seis Sigma tem promovido mudanças e benefícios significativos, a saber: aprimoramento da qualidade; conquista da satisfação dos clientes através da melhor compreensão dos requisitos exigidos; pleno entendimento das entradas críticas dos processos, necessárias para responder às alterações nas exigências e especificações definidas; ganhos no fluxo do processo; aumento da produtividade; redução de tempos de ciclo; aumento da capacidade produtiva e da confiabilidade dos produtos; redução de desperdícios e de atividades que não agregam valor ao processo; e maximização dos lucros. Em empresas de grande porte é notória a acentuada ênfase no uso de estatística para obtenção dos benefícios supracitados (ANDRIETTA; MIGUEL, 2007; PINT0 et al. 2008).

Tratando-se mais especificamente dos requisitos essenciais já mencionados, o conceito de qualidade ganhou uma conotação diferenciada com a inclusão de valor econômico e utilidade prática, tanto para o cliente como para a organização, cuja denominação de valor é percebida por ambos em cada aspecto da relação de negócio (HARRY; SCHROEDER, 2000). Esta conceituação de qualidade enfatiza aspectos pouco explorados com tanta ênfase até então por outras iniciativas de melhoria da qualidade. Além disso, reconhecer que há uma correlação direta entre o número de defeitos, o custo do desperdício e o grau de satisfação do cliente e aliar o padrão sigma de medir defeitos como métrica universal que pode ser aplicada a diferentes processos, simplificou a avaliação do desempenho em diferentes processos, independentemente da área funcional (HARRY, 1998; HARRY; SCHROEDER, 2000).

A literatura sugere que o Seis Sigma combina a abordagem estatística e a abordagem estratégica. A abordagem estatística está alicerçada na aplicação do pensamento estatístico, em que a priorização do foco em processos, o entendimento da variação e o uso de dados para subsidiar ações devem ser entendidos como componentes-chave nas atividades diárias de gerenciamento de processos (SANTOS, 2006). A preocupação contínua com a redução da variação, com a medição e com a coleta de dados são premissas indiscutíveis do Seis Sigma que se apoiam na metodologia científica. Compreender isso é primordial para que projetos sejam implementados com êxito e tragam o retorno esperado, tanto em retenção de conhecimento como em resultados financeiros.

Com foco na oportunidade de ganhos financeiros tangíveis, as organizações aproximam a implementação do Seis Sigma dos assuntos estratégicos, de modo que os objetivos e metas estratégicas passam a definir diretrizes para o programa. As metas são atingidas com a implementação de projetos que priorizam as diretrizes e políticas definidas estrategicamente. 0 programa é preponderantemente top-down. 
0 Seis Sigma está focado no gerenciamento por processos e por diretrizes e visa um avanço no grau de satisfação dos clientes e uma evolução qualitativa dos processos críticos. Além disso, a redução de desperdícios e da variabilidade nos processos produtivos são objetivos primários do programa. Alcançá-los possibilita um incremento importante para abater os investimentos da implementação e para alavancar sua sustentação.

Uma das formas de enxergar o porquê do Seis Sigma estar compreendido entre os assuntos estratégicos prioritários é observar seu potencial de programa estratégico, ou seja, capaz de determinar o impulso e a viabilidade global da entidade, bem como relacionar os processos de nível estratégico aos processos de suporte e operacionais (QUINN; MINTZBERG, 1991; ARMISTEAD et al., 1999). Para Ansoff e Mcdonnell (1993), um programa estratégico é responsável pelo potencial futuro dos lucros da organização. Tais propriedades fazem com que o Seis Sigma tenha um impacto tanto na formulação, como na implementação da estratégia competitiva da organização. De fato, compreendido dessa maneira, e sendo um programa de qualidade, o sucesso de sua implementação é obtido se houver um plano de ações para melhoria da qualidade que seja desenvolvido e ajustado à orientação estratégica (SANTOS, 2006).

A Figura 1 explicita essa ideia e sugere que o programa Seis Sigma esteja realmente inserido no contexto da gestão da qualidade e da gestão estratégica como um programa amplo que contempla atividades de planejamento, medição e priorização de ações de melhoria. Os projetos são derivados da ação e dinamismo do ambiente (de seus agentes diretos) sobre a orientação estratégica e missão, que direcionam a formulação da estratégia competitiva e a definição dos objetivos e metas estratégicos e operacionais.
No campo metodológico, a discussão sobre as contribuições do Seis Sigma, apoiada na forma prescritiva e sistematizada de aplicação do ciclo de melhoria DMAIC (Definir, Medir, Analisar, Incrementar e Controlar), dividiu pesquisadores acadêmicos. Opositores argumentam sobre os pressupostos estatísticos que justificam o padrão $6 \sigma$ e os 3,4 DPMO - defeitos por milhão de oportunidades; ressaltam o pouco avanço metodológico do DMAIC em relação ao ciclo PDCA idealizado por W. Shewart; duvidam da demonstração dos ganhos financeiros como decorrência de oportunidades de impacto direto na satisfação dos clientes; questionam a necessidade da estrutura tipo "receita de bolo" do DMAIC para aplicação de ferramentas já consagradas no contexto da gestão da qualidade; entre outras colocações (MONTGOMERY, 2004; MITRA, 2004; RAISINGHANI, 2005).

Por outro lado, deve-se salientar que o Seis Sigma introduz a aplicação do conhecimento sobre planejamento, modelagem e simulação de processos, controle e otimização integrados com conhecimentos estatísticos. Além disso, a sistematização do DMAIC permite monitorar e definir um método de diagnóstico que guia os engenheiros de processo a encontrarem oportunidades de ganhos significativos em processos e de aumento da lucratividade, em decorrência do enfoque em projetos criteriosamente selecionados (HAN; LEE, 2002).

No Seis Sigma, os valores ressaltados por Walter Shewart, William Edwards Deming e Kaoru lshikawa, são reforçados em vários aspectos com uma apresentação de conteúdo mais sistematizada e prescritiva. Isto não havia sido proposto anteriormente. A sistematização metodológica reforça e preserva também certos valores imprescindíveis do TQM (Total Quality Management), como: foco em processo; foco no cliente; comprometimento dos gestores; decisão

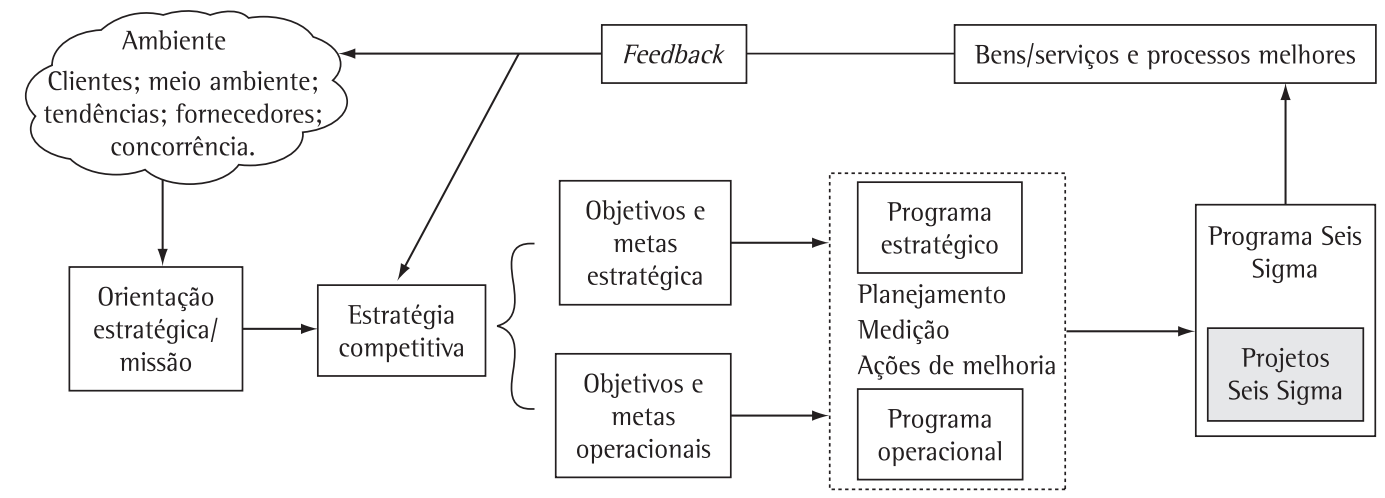

Figura 1. Seis Sigma como programa estratégico e operacional que contempla atividades de planejamento, de medição e de priorização de ações de melhoria. 
baseada em dados e fatos; melhoria contínua; e participação e comprometimento de todos. São valores da gestão da qualidade total sustentados pelo Seis Sigma (KLEFSJÖ et al., 2001).

A formação e capacitação de uma equipe de especialistas com identificação própria (master black belts, champion, black belts e green belts) para compor um grupo dos profissionais mais aptos para desenvolverem e promoverem o crescimento do desempenho do negócio, aplicando métodos e técnicas estatísticas para implementarem projetos definidos e/ou direcionados estrategicamente, é um ponto forte deste assunto. A existência de parte desses especialistas dedicados integralmente à melhoria da eficiência de alguns processos críticos do negócio acabou mudando o perfil do profissional da área de qualidade e reforçou a comunicação interfuncional (HOERL, 1998; INGLE; ROE, 2001).

Neste contexto, conforme discutido por SANDERS; HILD (2000), a forma de treinamento da equipe de belts, os critérios de escolha e os requisitos impostos para certificação são fatores que dependem da estratégia de desdobramento adotada pela organização. Com o comprometimento dos executivos líderes, tais investimentos para implementação do Seis Sigma são ratificados, reforça-se o cumprimento de metas e há mais garantia para o desdobramento e disseminação da cultura nos níveis gerencial e operacional. Pelo estudo de Kumar (2007), o comprometimento dos gestores é o fator crítico de sucesso de maior importância para o sucesso do Seis Sigma em empresas de pequeno e médio porte.

0 gerenciamento de projetos nos moldes do Seis Sigma deu mais foco para as atividades e possibilitou um maior comprometimento das pessoas pelo fato de os projetos serem formalizados por meio de contrato celebrado entre membros de uma equipe composta por profissionais de níveis hierárquicos e funções diferenciadas (ROTONDARO, 2002). É uma abordagem que se distingue da visão usual baseada em modelos que direcionam sobre "o que" deve ser feito para o sucesso do projeto. No Seis Sigma há maior percepção sobre "como" implementar os projetos para que sejam bem sucedidos (SCHROEDER et al., 2002) e, portanto, o gerenciamento de projetos vai além do aperfeiçoamento das atividades em áreas de conhecimento vinculadas a processos gerenciais, como sugerido pelo PMI - Project Management Institute (2004).

Goh (2002) aponta que a implementação dos projetos Seis Sigma está atrelada a problemas interfuncionais e multidisciplinares, e embutem uma forma fértil de se gerar e enriquecer ideias e técnicas, tendo o pensamento estatístico como um denominador comum. Usar uma abordagem orientada para dados e projetos é uma característica fundamental que aumenta a habilidade de reconhecer, descrever, gerenciar, controlar e mesmo utilizar a variação existente nos diversos processos.

Ainda neste contexto, Santos e Martins (2005) esclareceram uma das facetas da ligação entre tático e estratégico na definição dos projetos Seis Sigma ao identificarem que a medição de desempenho e o alinhamento estratégico são requisitos para o sucesso do Seis Sigma. Isto enfatiza a necessidade de os projetos serem direcionados estrategicamente e de darem um feedback para o sistema de medição de desempenho multidimensional.

\section{Método de pesquisa}

A obtenção dos subsídios empíricos da pesquisa embasou-se no método do estudo de caso (múltiplos casos) como procedimento de coleta de dados. Essa abordagem de pesquisa qualitativa foi adotada, considerando que não há controle por parte dos pesquisadores sobre os eventos abordados, devido à sua participação apenas de forma passiva (CRESWELL, 1994; BRYMAN, 1989; YIN, 2005). Além disso, é uma forma de relatar aspectos pertinentes sobre a implementação do programa Seis Sigma nas organizações, que é um tema de caráter contemporâneo, cuja relevância vem sendo corroborada na literatura em trabalhos que se valeram deste mesmo método de pesquisa para obtenção de subsídios empíricos (MACDAM; LAFFERTY, 2004; REVERE; BLACK, 2003; MOTWANI et al., 2004).

A seguinte questão de pesquisa motivou a realização dos estudos de caso: Como os investimentos no Seis Sigma afetam as ações gerenciais e estratégicas para que a implementação e estruturação seja efetiva em ambientes de manufatura e/ou serviços?

A coleta dos dados foi feita no ano de 2005 por meio de entrevistas semiestruturadas, nas quais especialistas black beltse green belts foram as fontes de evidências principais. De forma complementar, foram inseridas informações decorrentes de observações diretas realizadas pelos pesquisadores, durante as visitas às empresas.

Um protocolo de pesquisa foi elaborado para direcionar as entrevistas. Este incluiu 40 perguntas abertas, formuladas para melhorar a compreensão sobre a relevância dos requisitos essenciais discutidos em âmbito teórico.

Sobre a seleção das empresas, utilizou-se de escolha intencional com base em certos critérios, 
a saber: estágio da implantação do programa (procurou-se uma organização em estágio inicial e outra em estágio mais avançado); diferença quanto ao setor de mercado (uma de serviços e outra de manufatura); disposição para colaborar com a pesquisa (black belts se propuseram a conceder a entrevista e forneceram as evidências); facilidade de acesso por parte dos pesquisadores às fontes de evidências (proximidade geográfica do objeto de estudo e sucesso no contato prévio com os entrevistados); e similaridade potencial compatível com os casos descritos na literatura (buscaram-se empresas de grande porte com atuação em outros continentes). As duas subsidiárias brasileiras de multinacionais americanas estudadas que satisfizeram os referidos critérios são empresas com destaque no mercado nacional. Aqui estão identificadas com os nomes fictícios de TTFix e Fastcard.

Para facilitar o registro das informações e aumentar a confiabilidade dos resultados, as entrevistas foram gravadas, em seguida transcritas e preparadas para a análise dos resultados. As informações foram organizadas por tópico em consonância com a discussão teórica. Sobre a análise, deve-se ressaltar que não houve objetivo de comparar as duas empresas. Procurou-se, principalmente, mostrar que o Seis Sigma se diferencia de outros programas; requer gerenciamento efetivo, independente do setor de mercado; e exige investimentos para construção de uma infraestrutura apropriada em nível metodológico, tecnológico, cultural, de capacitação de pessoas e de definição de metas financeiras a médio e longo prazo que contribuem para o aumento da lucratividade e da produtividade.

\section{Detalhamento sobre o Seis Sigma nas empresas estudadas}

A primeira empresa (TTFix) integra um grupo empresarial americano cujas operações envolvem produção de elementos de fixação para o setor metal mecânico (indústria de autopeças). Suas operações no Brasil estão voltadas para o fornecimento de componentes utilizados em sistemas de fixação de automóveis. Líder de mercado, com uma fatia de 25\% do total negociado no mercado nacional em elementos de fixação, trabalha com um contingente de 550 funcionários distribuídos em duas fábricas e declara um faturamento em torno de US\$ 35 milhões decorrentes de transações com clientes como a Ford ${ }^{\circledR}$, Fiat $^{\circledR}, \mathrm{GM}^{\circledR}$, Volkswagen $^{\circledR}, \mathrm{Honda}^{\circledR}$, TRW ${ }^{\circledR}$, entre outras. Sua missão é se tornar a maior fornecedora de soluções de sistemas de fixação.
Investindo no Seis Sigma, a companhia provocou uma mudança estratégica que priorizou, preliminarmente, a redução da variabilidade e a identificação dos processos defeituosos. Mas essa abordagem não foi suficiente para ganhar competitividade imediata. Assim, a organização promoveu um enxugamento das operações em geral (eliminação de desperdícios). Com isso, a companhia pôde iniciar uma mudança cultural para que as prioridades estratégicas se viabilizassem, apesar da resistência inicial de mudar a cultura e quebrar paradigmas.

Em termos de benefícios imediatos, o Seis Sigma propiciou: a unificação da corporação; definição de prioridades estratégicas a longo prazo, voltando-se para a excelência e para o desenvolvimento de processos em nível de classe mundial; maiores investimentos em inovação; melhoria do gerenciamento do portfólio; desenvolvimento das pessoas; enfoque mais acentuado no mercado e no cliente; e o fortalecimento da marca.

Implementar o programa contribuiu para uma maior integração entre as pessoas. Na verdade, a TTFix já tinha propostas nesta linha, pois a gestão por processos é um dos requisitos da TS-16949 já implementada. Mas, apesar disso alguns aspectos culturais previamente instituídos que ressaltavam a integração em algumas áreas (área Comercial, por exemplo), ficaram ainda mais carentes de integração com o Seis Sigma.

A disciplina e clareza de propósitos para as ações foram mais bem esclarecidas com o Seis Sigma. As pessoas sentiram-se mais conscientes de seus papéis; ficou mais evidente a expectativa sobre a contribuição de cada membro da equipe; e a forma de obtenção dos resultados mais explicitada. Com isso, o Seis Sigma formalizou a comunicação e o entendimento entre as pessoas, e gerou a expectativa de que ninguém se disporia a trabalhar num projeto de melhoria ou na solução de um problema sem considerar o trabalho em equipe.

Do ponto de vista metodológico, a TTFix definiu a estrutura do programa embasando-se no Lean Manufacturing (manufatura enxuta), no DMAIC e no DFSS (Design for Six Sigma). As duas primeiras direcionadas para a melhoria e a última voltada para a inovação.

No concernente à formação e capacitação dos especialistascomo parteprimordialdaimplementação, vale destacar algumas especificidades acerca dos cursos que são ministrados. No treinamento dos black belts, há forte ênfase em teoria estatística, porque ela é utilizada como ferramenta para solução de problemas. 0 aprendizado das ferramentas do 
DMAIC e do DFSS tem atenção prioritária, mas também se enfatizam os princípios de manufatura enxuta, os quais incluem o estudo dos capacitadores que englobam as tecnologias, metodologias e as ferramentas empregadas na manufatura enxuta (GODINHO FILHO; FERNANDES, 2004). Assim, seguem o modelo Lean Six Sigma.

A abordagem estratégica é menos enfatizada pelo fato de que, na visão da companhia, o enfoque estratégico deve entrar na hora de selecionar os projetos, para se ter a garantia de que estes realmente fiquem alinhados aos objetivos estratégicos da empresa.

Com relação ao treinamento aplicado pelos black belts aos green belts, os procedimentos definidos são: estimular a equipe; gerar maior envolvimento e comprometimento de todos com o projeto, impulsionados pelas atividades de Kaizen; utilizar vocabulário simples e linguagem mais acessível (sem palavras ou expressões em inglês); interação constante e busca de feedback sobre o grau de compreensão dos conceitos; listar diariamente as principais dúvidas e dificuldades para dirimi-las o quanto antes; utilizar situações do cotidiano da equipe para exemplificar a utilização das ferramentas estatísticas. Em três anos, a TTFix compôs uma equipe de especialistas com três black belts, nove green belts e vinte e cinco especialistas em Kaizen.

Dentre os diversos assuntos relacionados à implementação do Seis Sigma, a definição dos projetos figura como um ponto de particular atenção, sendo um assunto de cunho estratégico de competência do gerente geral da fábrica. Este gerente, também champion, é a pessoa que ocupa a posição mais elevada da fábrica. É ele quem tem as metas de resultados passadas pela matriz. Além disso, ele define a área que requer melhoria e qual a meta de resultado almejada. 0 master black belt, o black belt e o champion são os encarregados pelo delineamento do escopo dos projetos.

Com o conhecimento preliminar sobre o escopo do projeto relacionado ao processo crítico que requer melhoria, o black belt decide se ele se encarregará sozinho de desenvolver o projeto ou se engajará algum green belt na tarefa, já que o green belt participa dos projetos sempre sob a supervisão do black belt. Caso algum green belt seja incluído na tarefa, ele terá a responsabilidade de traçar um primeiro esboço do escopo, o qual será posteriormente revisado pelo black belt.

Para definição do escopo, o black belt deve detalhar uma série de itens. Dentre eles: (1) Definir objetivos; (2) Descobrir se o projeto está alinhado com os quatro alavancadores do negócio (resultado líquido, satisfação do cliente, retorno sobre investimento e fluxo de caixa); (3) ldentificar quais são as restrições para melhoria, quando houver; (4) Estabelecer limites, identificando o que fica dentro do problema e o que fica fora. Em particular, é mais importante saber o que fica fora dos limites, ou seja, a parte que não será alterada com a melhoria proposta; (5) Quantificar os ganhos financeiros, baseando-se em informações asseguradas por um profissional da área financeira da empresa. Esse profissional avaliará o tipo de ganho almejado quanto ao seu impacto em termos de ganho imediato (receita direta) ou ganho potencial (oportunidades); e (6) Definir os papéis dos patrocinadores do projeto, incluindo a presença do vice-presidente executivo de projetos Seis Sigma, do master black belt, do black belt, do champion, do supervisor da área funcional, e do analista financeiro. Este último atesta a viabilidade financeira do projeto e quantifica o resultado.

Em termos conceituais, a TTFix trabalha com a ideia básica de que um projeto Seis Sigma está associado sempre ao grau de dificuldade de solução do problema e da necessidade da melhoria propiciar ganhos permanentes ou de longa duração, ou seja, de que é um projeto que permite soluções que sustentam os ganhos financeiros por muito tempo. Em função disso, a abordagem Seis Sigma não costuma ser utilizada de imediato, mas sim, como uma última tentativa de se solucionar definitivamente o problema.

0 ajuste do escopo dos projetos é uma tarefa estrita do black belt, tendo em vista que sua meta mínima de desempenho é um ganho de pelo menos 1 sigma; o que significa uma melhoria no processo de 70\%. Ao definir o escopo, o black belt deve verificar se o projeto proposto está alinhado aos alavancadores do negócio. Estes alavancadores darão o direcionamento para a definição dos projetos Seis Sigma e visam impulsionar os seguintes pontos: (1) Construção da marca (satisfação do cliente; crescimento orgânico; taxa de captura de novos negócios); (2) Desenvolvimento de pessoas; (3) Gerenciamento do portfólio e excelência corporativa (aqui entram indicadores de desempenho associados ao Seis Sigma); e (4) Finanças (retorno sobre investimento; fluxo de caixa; lucro operacional líquido; vendas por empregado, e outros).

Para auxiliar no gerenciamento dos projetos Seis Sigma, a organização dispõe de um sistema computacional que é operado via Web na intranet. Este sistema contém dados sobre cada projeto em suas diferentes fases de implementação. Dados são inseridos pelas áreas financeiras de cada fábrica com o propósito de facilitar o acompanhamento do status 
dos projetos, do controle gerencial dos resultados financeiros dos projetos e informações sobre os responsáveis. Este sistema contribui sobremaneira para a eficiência gerencial do programa na TTFix.

A empresa Fastcard faz parte de um grupo empresarial de origem norte-americana que opera no setor de serviços, efetuando transações financeiras, comercializando cartões de crédito e provendo serviços de turismo e viagens de negócios.

A subsidiária brasileira da Fastcard, que atua no segmento de cartões de crédito, tem um dos mais modernos centros de operações da América Latina no interior de Minas Gerais. Cerca de 1,2 mil funcionários trabalham essencialmente com serviços relacionados aos cartões de compra e crédito, cheques de viagem e serviços corporativos. Os clientes da Fastcard são portadores de cartão de crédito, tanto pessoas físicas como pessoas jurídicas e estabelecimentos que aceitam seu cartão. A Fastcard é uma das poucas do setor que faz a gestão dos dois grupos de clientes (portadores e estabelecimentos) e tem o cartão de crédito corporativo como um de seus produtos mais fortes.

No setor de serviços, uma das características principais é que a relação com o cliente é bastante volátil e o feedback desses clientes é mais rápido do que aconteceria numa indústria de manufatura. Em entrevista, o black belt, mencionou que a expectativa do cliente na indústria de serviço é muito mais forte; o que faz com que ele tenha mais poder de influenciar na performance do que teria num ambiente de manufatura. Além disso, o cliente fornece um constante feedback instantâneo sobre a efetividade do produto.

A alta concorrência e a dificuldade de manter a fidelidade à marca são pontos que caracterizam o negócio. Por esta razão, a Fastcard vale-se do Seis Sigma como estratégia de diferenciação para ganhar vantagem competitiva. Frente ao fato de que o preço não é um objetivo de desempenho primário para conseguir aumentar o número de clientes, a Fastcard tenta se diferenciar dos concorrentes recorrendo a métodos que reduzam a ocorrência de defeitos e falhas e que promovam uma melhoria da qualidade dos serviços que seja percebida pelos clientes. Neste sentido, a Fastcard procura a fidelidade de seus clientes por meio do aumento do valor agregado de seus produtos.

Três fases caracterizam a implementação do Seis Sigma na Fastcard. A fase inicial é a de implantação do programa. Ela ocorre por meio do treinamento dos especialistas (green belts, black belts e master black belts), os quais constituem a fundação, o alicerce necessário para começar a promover qualquer melhoria com base nos conceitos do Seis Sigma. Na segunda fase, os especialistas treinados são estimulados fortemente a conseguirem suas certificações para que possam planejar e gerenciar as ações corretivas nos processos; e para identificarem os projetos destinados à correção de processos defeituosos, tendo em mente a preocupação constante de aumentar a satisfação dos clientes. A terceira fase consiste em começar a desenhar novos processos com suporte da metodologia Seis Sigma.

0 enfoque estratégico é marcante. Os métodos estatísticos são meios para se conseguir a eficiência necessária no gerenciamento dos processos. Inserida nesse foco principal está a satisfação do cliente, e sobre ela deve ser observado que um defeito é identificado no momento em que o cliente faz algum tipo de solicitação ou reclamação decorrente de sua insatisfação pelo fato de não poder ser atendido pelos procedimentos operacionais de rotina no tempo que ele gostaria.

0 desdobramento estratégico é fundamentado no estudo dos processos críticos que impactam mais os clientes, os quais são: desenvolvimento de novos produtos, serviços e relacionamentos; obtenção de novos clientes; processamento das transações; processamento de extratos, pagamentos e reconciliações; atendimento a clientes; entre outros.

Em termos metodológicos, a aplicação de ferramentas para melhoria da qualidade visa diferenciação e aumento do potencial competitivo. A abordagem estatística do Seis Sigma enfoca os quatro processos-chave que impactam diretamente os clientes. São eles: (1) Pagamento de clientes; (2) Pagamento aos estabelecimentos; (3) Autorizações e despesas; e (4) Aquisição de cartões.

A estrutura de implementação básica dos projetos de melhoria contempla o DMAICL, que consiste nas fases do DMAIC acrescidas de uma última (Leverage). Esta última etapa da metodologia constitui uma obrigatoriedade do resultado alavancar outros processos além daquele que está sendo corrigido. O DFSS é empregado no desenho de novos processos e na otimização de processos já existentes. SSPM (Six Sigma Process Management) é outra metodologia para gerenciamento de processos que usa a fundamentação conceitual do Seis Sigma. Mais recentemente foi introduzido um módulo sobre manufatura enxuta no treinamento dos especialistas.

Assim como em qualquer ambiente industrial, na indústria de serviços, vários fatores podem comprometer o sucesso da implementação das estratégias adotadas para suplantar a concorrência. Em particular, quando se fala em serviço, a relação 
com as pessoas que fornecem o serviço é imediata. Assim, para conseguir implementar com sucesso o Seis Sigma, é imprescindível haver uma identificação precisa dos gestores primários. Logo de início, um passo é identificar um ou mais gestores que tenham conhecimento suficiente sobre os processos críticos para que seja dado o suporte necessário para o black belt gerenciar um processo por inteiro. A ideia não é fazer com que este gestor aceite a metodologia, mas sim, que ele enxergue os benefícios de sua aplicação e compreenda a necessidade de utilização dos métodos.

A identificação dos gestores secundários também foi fundamental. São pessoas que atuam como gerentes de departamento e estão mais ligados aos vários procedimentos relacionados com partes dos processos afetados pela melhoria. São eles que facilitam e tornam disponíveis os recursos necessários para que os projetos sejam implementados e gerenciados.

A definição clara dos objetivos estratégicos do negócio, aliada ao comprometimento desses gestores com a filosofia Seis Sigma e a existência de uma equipe de especialistas qualificados formaram um tripé fundamental para o sucesso do Seis Sigma na Fastcard. Como reflexo desse sucesso, a empresa em cinco anos conseguiu resultados sustentáveis a médio e longo prazo; melhorou o padrão de qualidade de seus serviços, proporcionando um retorno em termos de receita para a empresa também a médio e longo prazo; gerou mais satisfação dos funcionários (as reclamações diminuíram e eles passaram a se preocupar com outras tarefas que não apenas corrigir defeitos ou falhas); e conseguiu oferecer aos clientes um serviço de maior qualidade e um produto de maior valor agregado. Em cinco anos, a empresa treinou vinte black belts e quarenta e cinco green belts.

$\mathrm{Na}$ Fastcard, a preocupação com a redução das falhas operacionais é constante. Por isso, para sua certificação, cada black belt deve implementar um projeto que gere uma redução de $80 \%$ na quantidade de defeitos. Além disso, como na Fastcard o alinhamento estratégico é um componente essencial para a definição dos projetos Seis Sigma, em geral, a definição dos projetos seguem os objetivos estratégicos que têm maior impacto no cliente. lsto significa que algumas medidas de desempenho do negócio relacionadas aos clientes são direcionadoras de projetos. Assim, apesar de todos seguirem um alinhamento estratégico, alguns projetos Seis Sigma são influenciados mais fortemente pela estratégia que é definida anualmente pela unidade de negócio. Neste caso, os projetos Seis Sigma servem de direcionadores estratégicos; o que demonstra mais claramente a eficiência da solução encontrada com sua implementação.

Para gerenciamento dos projetos, a Fastcard dispõe de um sistema de controle para acompanhamento da implementação que funciona localmente em cada unidade de negócio. Além disso, há também outro sistema corporativo que é operado via Web para acompanhar e controlar o desempenho do negócio à luz dos resultados do Seis Sigma. Este sistema de gerenciamento do desempenho do programa Seis Sigma é denominado de Repositório Mundial de Projetos e de Resultados, e tem como finalidade possibilitar ao Six Sigma Council gerir os resultados provenientes dos projetos concluídos ou em fase de implementação. Ele funciona também como um centro de informações para manter os black belts sintonizados uns com os outros, acompanhando os avanços conseguidos por pessoas de outras localidades; observando o retorno sobre o investimento em cada projeto implementado; e permitindo uma integração maior entre as equipes de especialistas.

Ainda no âmbito da implementação dos projetos, uma das metas principais relativa ao gerenciamento dos processos é tornar os processos mais robustos. Como na maior parte dos processos há intervenção humana, a empresa salienta o treinamento, a comunicação e a disseminação da cultura Seis Sigma em todos os níveis de atividades. Em alguns casos, as oportunidades de melhoria surgem a partir das causas especiais de variação nos processos. Todavia, o que prevalece na seleção de projetos é o quanto uma oportunidade de melhoria pode impactar o cliente. Em outras palavras, um projeto Seis Sigma deve, a rigor, seguir o alinhamento estratégico para que tenha suporte gerencial em sua implementação.

\section{Análise dos resultados}

No que se refere à razão para os investimentos, verificou-se que para alcançar a mudança de orientação estratégica, melhoria de processos ou resultados sustentáveis tanto a TTFix como a Fastcard tiveram que fazer vultosos investimentos, principalmente, em relação à formação das pessoas e à criação de uma infraestrutura tecnológica que facilitasse a implementação e o gerenciamento dos projetos. Conforme a Tabela 1, que complementa as informações da pesquisa de campo e traz características sobre o desdobramento do Seis Sigma nas organizações estudadas, a melhoria da qualidade e redução de desperdícios, na TTFix, e a melhoria da qualidade dos serviços e aumento da satisfação do cliente, na Fastcard, foram objetivos primários 
para o investimento. Tais objetivos reforçam que 0 investimento numa gestão quantitativa tem reflexos significativos sobre a mudança de cultura, a qual é um dos investimentos intangíveis a médio e longo prazo do Seis Sigma, além de consistir num dos cinco fatores críticos de sucesso mais importantes do Seis Sigma para empresas de qualquer porte (KUMAR, 2007).

Em termos de abordagens, as evidências empíricas revelam que é preciso ir além da abordagem estatística para se ter sucesso com o Seis Sigma. Além da efetividade operacional, a abordagem estratégica é fundamental, em particular, na definição e seleção dos projetos. Verificou-se que o foco dos projetos condiz com uma orientação estratégica voltada para o mercado, no qual o impacto no cliente e o uso dos indicadores de desempenho do negócio são primordiais na definição dos projetos.
Tanto na TTFix como na Fastcard, o Seis Sigma se caracteriza por ser um programa estratégico implementado em nível corporativo e gerenciado por líderes com posição estratégica na organização. Ambas as empresas adotaram a estratégia de desdobramento denominada por Sanders e Hild (2000) como "Organização de Engenharia Seis Sigma".

Investimentos em sistemas computacionais próprios que incrementem a integração das atividades e a comunicação refletem o esforço para que a disciplina e o comprometimento sejam mantidos. Esse recurso tecnológico fornece uma infraestrutura apropriada para acompanhar e gerenciar o status dos projetos, o desempenho das equipes, entre outros pontos que revelam a "força" do programa Seis Sigma na organização como um todo.

Tabela 1. Características típicas do Seis Sigma na TTFix e na Fastcard.

\begin{tabular}{lccc}
\multirow{2}{*}{ Característica } & \multicolumn{3}{c}{ Organização } \\
\cline { 2 - 4 } & & TTFix & Fastcard \\
\hline Tempo da & 3 anos & 5 anos &
\end{tabular}

Razão do investimento no Seis Sigma

Forma de implementação e objetivos primários do Seis Sigma

Requisitos para ser black belt

Impacto dos

Projetos Seis Sigma

Sistema de controle gerencial

Fase de implementação

Ganhos financeiros
Mudança de orientação estratégica; melhoria dos processos; e excelência em gestão.

Programa corporativo que visa melhoria da qualidade dos processos e redução de desperdícios.

Ser selecionado pela alta administração; possuir capacidade de liderança; ter visão geral do negócio e da corporação; atuar em tempo integral; aplicar as ferramentas da metodologia Seis Sigma (DMAIC e DFSS) e de manufatura enxuta; liderar times; treinar e orientar green belts, e conduzir projetos que somam US $\$ 250$ mil em economias com

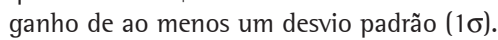

São direcionadores de desempenho; devem impactar os alavancadores do negócio; são focados em resultados financeiros e em redução de defeitos e de custos de produção.

0 sistema de controle gerencial (Power Steering ) é corporativo e destina-se ao acompanhamento do status dos projetos e das equipes responsáveis. 0 sistema é operado via Web na intranet da empresa, e é gerenciado pela alta administração corporativa.

Inicial (estágio de capacitação das pessoas e disseminação da cultura).
Obtenção de resultados sustentáveis.

Programa corporativo que visa melhoria da qualidade dos serviços e a satisfação do cliente.

Deve ter experiência com o gerenciamento de projetos e de processos; ter perícia em análise de dados e de situações; ser engenheiro; aplicar as ferramentas da metodologia Seis Sigma (DMAICL e DFSS); liderar equipes de green belts; gerenciar processos de ponta a ponta; e atuar como consultores internos em questões relativas à análise de dados.

São direcionadores de desempenho; devem impactar diretamente o cliente e melhorar processos críticos; são focados em qualidade, redução de falhas, e aumento da capacidade dos processos.

O sistema de controle gerencial (Repositório mundial de projetos e resultados) é corporativo e visa o gerenciamento dos projetos e da equipe envolvida. 0 sistema é operado via $W e b$ na intranet da empresa, e é gerenciado pelo Six Sigma Council.

Avançada (preocupação com o aprimoramento da metodologia e aumento do número de especialistas).

Não informado 
Sobre o aspecto metodológico, nota-se que, talvez por ser uma empresa de manufatura, a TTFix aparenta estar mais consciente da contribuição dos conceitos de manufatura enxuta como metodologia efetiva de combate ao desperdício e a variação. 0 esforço em melhorar continuamente, procurando a remoção de perdas e desperdícios é um princípio do pensamento enxuto que reforça os propósitos do Seis Sigma. Neste sentido, conforme aponta Arnheiter e Maleyeff (2005), uma vez que o desempenho do negócio é determinado por interações complexas de pessoas, materiais, equipamentos e recursos financeiros, então, uma organização que capitalize a força do Seis Sigma com os conceitos da manufatura enxuta, ou até melhor, da gestão enxuta, corre menos risco de falhar no propósito de atingir um nível de excelência absoluta. A proposta das empresas de combinar os princípios de Lean manufacturing ao Seis Sigma (origem do Lean Sigma ou Lean Six Sigma) reforça a tendência recente das discussões sobre o tema na literatura (BYRNE et al. 2007; GEORGE, 2004; NASLUND, 2008). Neste sentido, black belts consideraram que essa combinação reduz o tempo médio de implementação dos projetos, mas seria difícil estimar o quanto do sucesso deles se deve a uma ou outra abordagem.

Em termos de formação e capacitação das pessoas, deve ser ressaltada a importância do comprometimento e da disseminação da cultura, os quais, aliados à visão dos especialistas, impulsionam o sucesso do Seis Sigma, seja para uma empresa de manufatura ou de serviços. Neste sentido, os perfis especificados na Tabela 1 para os black belts ratificam essa importância. Além disso, não permitir a perda de interesse das pessoas pela conclusão dos projetos; viabilizar a execução dos projetos em todas as suas fases; e avaliar o que os projetos agregam em retenção de conhecimento são expectativas observadas atentamente pelos executivos líderes.

$\mathrm{Na}$ Fastcard, foi verificado que o crescimento do desempenho do negócio é mais dependente de fatores comportamentais. Por essa razão, as pessoas talvez sejam o componente que mais compromete o sucesso da implementação do programa e não a falta de metodologia eficiente para analisar dados de processo. Evidentemente, que a formação de massa crítica sobre os conceitos assegura o sucesso, mas como já apontou Dale et al. (2000): o Seis Sigma envolve mudanças profundas no modo de agir e pensar das pessoas; requer maturidade e maior grau de sofisticação da organização para controlar seus processos críticos com aderência e envolvimento das pessoas treinadas trabalhando em equipes interfuncionais; e deve priorizar uma constante preocupação com a redução de defeitos como um fator crítico para o cliente.

No que toca à definição e seleção dos projetos, a pesquisa revelou que: a necessidade de alinhamento estratégico e impacto no cliente são pontos fundamentais. À medida que se considere que a implantação do programa se inicia quando os projetos são direcionados, começam a ser implementados, os resultados aparecem efetivamente e proporcionam um feedback estratégico. Nas empresas estudadas, um projeto Seis Sigma não prioriza necessariamente resultados financeiros, embora estes sejam estimados quando o contrato é celebrado entre os membros responsáveis pelo projeto. Estes resultados decorrem de um conjunto de fatores, tais como: melhoria da qualidade; redução de defeitos e de causas de variação; redução de custo; identificação de oportunidades, impacto no cliente; mudança de cultura; necessidade de visão sistêmica dos processos, etc. Entre outros pontos, a Tabela 1 destacou que os projetos Seis Sigma servem como direcionadores de desempenho em ambas as organizações. 1sto reforça tanto a importância do uso dos indicadores de desempenho para aumentar o impacto dos projetos sobre os resultados, como a necessidade de um sistema de medição de desempenho dinâmico que forneça o suporte devido para que os projetos sejam selecionados.

Os resultados desta pesquisa dão indicativos mais seguros de que a própria concepção do que é um projeto Seis Sigma tem implicações diretas na forma de gerenciá-los e no nível de maturidade gerencial requerido para que sejam implementados efetivamente. 0 conhecimento, certificação e experiência dos gestores, baseados em modelos que valorizam o que deve ser feito, têm que ser complementados com o entendimento da estrutura metodológica do Seis Sigma que direciona como fazer. Sem essa visão mais completa corre-se o risco dos gestores subestimarem o uso da metodologia sistematizada, achando que ela agrega mais complexidade aos processos ou que seja indicada para situações cujas falhas gerem efeitos de alto custo.

\section{Considerações finais}

Os subsídios empíricos obtidos propiciaram considerar que as contribuições decorrentes dos investimentos no Seis Sigma estão atreladas a uma estrutura abrangente, porém, enxuta, planejada para ser implementada integralmente em um ambiente gerencial propício. Parece ilusório pensar em lucratividade apenas com o treinamento de alguns especialistas e com a implementação de projetos isolados. 0 retorno sobre os investimentos no 
Seis Sigma dificilmente acontece a curto prazo. 0 imediatismo, na maioria dos casos, apenas ressalta deficiências e desperdícios excessivos que estavam escondidos nos processos produtivos e não leva a solucionar problemas crônicos que demandam conhecer mais a fundo o potencial das ferramentas da metodologia sistematizada.

A expectativa de ganhos financeiros significativos a médio e longo prazo se concretiza mediante um efetivo gerenciamento dos projetos. Esse requisito essencialmente relevante dentre as contribuições do Seis Sigma inclui prerrogativas gerenciais em relação aos métodos tradicionais de engenharia da qualidade e engenharia econômica. Impor uma revisão rigorosa dos projetos com suporte de um master black belt; valorizar e priorizar o tempo/esforço para planejamento, visando uma implementação bem sucedida no menor tempo possível; e primar por um sistema de medição eficaz são as vantagens mais perceptíveis.

De acordo com as evidências, investir na implementação do Seis Sigma implica em mudanças mais profundas e abrangentes que afetam o capital intelectual da empresa e o modelo de gestão, valorizado pelo rigor e disciplina no uso de métodos quantitativos. Desde que fomentado pelas lideranças, projetos são sugeridos, a implementação é viabilizada, os resultados aparecem e a mudança cultural é gradativamente instalada. Em contrapartida, o viés financeiro como condição para o comprometimento dos gestores coloca em risco o progresso e o êxito do programa.

\section{Referências}

ANSOFF, H. 1.; McDONNELL, E. J. Implantando a administração estratégica. São Paulo: Atlas, 1993.

ANDRIETTA, J. M.; MIGUEL, P. A. C. Aplicação do programa seis sigma no Brasil: resultados de um levantamento tipo survey exploratório-descritivo e perspectivas para pesquisas futuras. Gestão e Produção, v. 14, n. 2, p. 203-219, 2007.

ARMISTEAD, C.; PRITCHARD, J. P.; MACHIN, S. Strategic business process management for organizational effectiveness. Long Range Planning, v. 32, n. 1, p. 96-106, 1999.

ARNHEITER, E.; MALEYEFF, J. The integration of lean management and six sigma. The TQM Magazine, v. 17, n. 1, p. 5-18, 2005.

ANTONY, J. Six Sigma for service processes. Business Process Management Journal, v. 12, n. 2, p. 234-248, 2006.

BEHARA, R. S.; FONTENOT, G. F.; GRESHAM, A. Customer satisfaction measurement and analysis using six sigma. International Journal of Quality and Reliability Management, v. 12, n. 3, p. 9-18, 1995.

BLAKESLEE JUNIOR, J. A. Implementing the six sigma solution. Quality Progress, v. 32, n. 7, p. 77-85, 1999.
BRETT; C.; QUEEN, P. Streamlining enterprise records management with lean six sigma. Information Management Journal, v. 39, n. 6, p. 58-62, 2005.

BRYMAN, A. Research methods and organization studies. London: Unwin Hyman, 1989.

BYRNE, G.; LUBOWE, D.; BLITZ, A. Using Lean Six Sigma approach to drive innovation. Strategy and Leadership, v. 35, n. 2 , p. 5-10, 2007.

CORONADO, R. B.; ANTONY, J. Critical success factors for the successful implementation of six sigma projects in organisations. The TQM Magazine, v. 14, n. 2, p. 92-99, 2002.

CHAKRABARTY, A.; TAN, K. C. The current state of six sigma application in services. Managing Service Quality, v. 17, n. 2, p. 194-208, 2007.

CRESWELL, J. W. Research design: qualitative and quantitative approaches. London: SAGE, 1994.

DOES, R. et al. Comparing nonmanufacturing with traditional applications of six sigma. Quality Engineering, v. 15, n. 1, p. 177-182, 2002.

GEORGE, M. L. Lean Six Sigma para serviços. Rio de Janeiro: Qualitymark, 2004. 436 p.

GODINHO FILHO, M.; FERNANDES, F. C. F. Manufatura enxuta: uma revisão que classifica e analisa os trabalhos apontando perspectivas de pesquisas futuras. Gestão e Produção, v. 11, n. 1, p. 1-19, 2004.

$\mathrm{GOH}, \mathrm{T}$. N. The role of statistical design of experiments in six sigma: perspectives of a practitioner. Quality Engineering, v. 14 , n. 4 , p. 659-671, 2002.

$\mathrm{GOH}, \mathrm{T}$.; XIE, M. Improving on the six sigma paradigm. The TQM Magazine, v. 16, n. 4, p. 235-240, 2004.

HAN, C.; LEE, Y. Intelligent integrated plant operation system for six sigma. Annual Reviews in Control, v. 26, n. 1, p. 27-43, 2002.

HARRY, M. J. Six sigma: a breakthrough strategy for profitability. Quality Progress, v. 31, n. 5, p. 60-64, 1998.

HARRY, M. J.; SCHROEDER, R. Six sigma: the breakthrough management strategy revolutionizing the world's top corporations. New York: Doubleday, 2000.

HOERL, R. W. Six sigma and the future of the quality profession. Quality Progress, v. 31, n. 6, p. 35-42, 1998.

INGLE, S.; ROE, W. Six sigma black belt implementation. The TQM Magazine, v. 13, n. 4, p. 273-280, 2001.

KLEFSJÖ, B.; WIKLUND, H.; EDGEMAN, R. L. Six sigma sees as a methodology for total quality management. Measuring Business Excellence, v. 5, n. 1, p. 31-35, 2001.

KUMAR, M. Critical success factors and hurdles to six sigma implementation: the case of a UK manufacturing SME. International Journal of Six Sigma and Competitive Advantage, v. 3, n. 4, p. 333-351, 2007.

McADAM, R.; BAlLIE, B. Business performance measures and alignment impact on strategy: the role of business improvement models. International Journal of Operations and Production Management, v. 22 n. 9, p. 972-996, 2002.

McFADDEN, F. R. Six-sigma quality programs. Quality Progress, v. 26, n. 6, p. 37-42, 1993.

MITCHELL, B. The Six Sigma appeal. Engineering Management Journal, p. 41-47, 1992. 
MITRA, A. Six Sigma education: a critical role for academia. The TQM Magazine, v. 16, n. 4, p. 293-302, 2004.

MONTGOMERY, D. C. Introdução ao controle estatístico da qualidade. 4 ed. Rio de Janeiro: LTC, 2004. 513 p.

MOTWANI, J.; KUMAR; ANTONY, J. A business process change framework for examining the implementation of six sigma: a case study of Dow Chemicals. The TQM Magazine, v. 16, n. 4, p. 273-283, 2004.

NASLUND, D. Lean, six sigma and lean sigma: fads or real process improvement methods?. Business Process Management Journal, v. 14, n. 3, p. 269-287, 2008.

NONTHALEERAK, P.; HENDRY, L. Exploring the six sigma phenomenon using multiple case study evidence. International Journal of Operations and Production Management, v. 28, n. 3, p. 279-303, 2008.

PANDE, P.; NEUMAN, R. P.; CAVANAGH, R. R. Estratégia Seis Sigma. Rio de Janeiro: Qualitymark, 2001.

PINTO, S. H. B; CARVALHO, M. M.; HO, L. L. Main quality programs characteristics in large size Brazilian companies. International Journal of Quality and Reliability Management, v. 25, n. 3, p. 276-291, 2008.

Project Management Institute - PMI. A guide to the project management body of knowledge. 2 ed. New York: Project Management Institute, 2004.

QUINN, J. B.; MINTZBERG, H. The strategy process: concepts, contexts, cases. New Jersey: Prentice Hall, 1991.

RAISINGHANI, M. S. Six sigma: concepts, tools, and applications. Industrial Management and Data Systems, v. 105, n. 4, p. 491-505, 2005.
ROTONDARO, R. G. Seis Sigma: estratégia gerencial para melhoria de processos, produtos e serviços. São Paulo: Atlas, 2002.

REVERE, L.; BLACK, K. Integrating six sigma with total quality management: a case example for measuring medication errors. Journal of Healthcare Management, v. 48, n. 6, p. 377-391, 2003.

SANDERS, D.; HILD, C. R. A discussion of strategies for six sigma implementation. Quality Engineering, v. 12, n. 3, p. 303-309, 2000.

SANTOS, A. B. Modelo de referência para estruturar o programa de qualidade Seis Sigma: proposta e avaliação. São Carlos, 2006. 312 p. Tese (Doutorado em Engenharia de Produção) - Universidade Federal de São Carlos UFSCar.

SANTOS, A. B.; MARTINS, M. F. A implementação dos projetos seis sigma contribuindo para o direcionamento estratégico e para o aprimoramento do sistema de medição de desempenho. In: SIMPÓSIO DE ADMINISTRAÇÃO DA PRODUÇÃO, LOGÍSTICA E OPERAÇÕES INTERNACIONAIS - SIMPOI, 7, 2005. Anais... (CD-ROM).

SCHROEDER, R. G. et al. Six sigma: old wine in new bottles? Minnesota: University of Minnesota, 2002.

SULLIVAN, L. P. Reducing variability: a new approach to quality. Quality Progress, v. 17, n. 7, p. 15-21, 1984.

TADIKAMALLA, P. R. The confusion over six-sigma quality. Quality Progress, v. 24, n. 11, p. 83-85, 1994.

YIN, R. K. Estudo de caso: planejamento e métodos. 3 ed. Porto Alegre: Bookman, 2005.

\title{
Contributions from Six Sigma: case studies in multinationals
}

\begin{abstract}
The literature suggests that Six Sigma has made many contributions to quality and strategic management. However, there is a certain lack of information about this subject in the Brazilian organizational scene. This paper aims to reduce this lack by means of a practical vision of the contributions that came from the investment of Brazilian companies in Six Sigma implementation. The relevance of essentials requirements, such as: quality concept; combination of statistical and strategic approaches; methodological systemizing; belt training; and project management is discussed from both the theoretical and practical aspects. Case study method was applied to obtain an empirical basis for the research. The investment in Six Sigma undertaken by two Brazilian branches of large multinationals (one a Manufacturing Company and the other a Service Company) are described and analyzed, underlining the relevance of these requirements.
\end{abstract}

\section{Keywords}

Six Sigma. Quality management. Six Sigma projects. Automotive industry. Credit cards. 